\title{
A dosimetric comparison of volumetric modulated arc therapy (VMAT) and non-coplanar intensity modulated radiotherapy (IMRT) for nasal cavity and paranasal sinus cancer
}

Yuri Jeong, Sang-wook Lee*, Jungwon Kwak, Ilsung Cho, Sang Min Yoon, Jong Hoon Kim, Jin-hong Park, Eun Kyung Choi, Si Yeol Song, Young Seok Kim, Su Ssan Kim, Ji Hyeon Joo and Seung Do Ahn

\begin{abstract}
Background: To compare dosimetric parameters of volumetric modulated arc therapy (VMAT) and non-coplanar intensity modulated radiotherapy (IMRT) for nasal cavity and paranasal sinus cancer with regard to the coverage of planning target volume (PTV) and the sparing of organs at risk (OAR).

Methods: Ten patients with nasal cavity or paranasal sinus cancer were re-planned by VMAT (two-arc) plan and non-coplanar IMRT (7-, 11-, and 15-beam) plans. Planning objectives were to deliver 60 Gy in 30 fractions to 95\% of PTV, with maximum doses ( $D_{\max }$ ) of $<50 \mathrm{~Gy}$ to the optic nerves, optic chiasm, and brainstem, $<40$ Gy to the eyes and $<10 \mathrm{~Gy}$ to the lenses. The target mean dose ( $\left.\mathrm{D}_{\text {mean }}\right)$ to the parotid glands was $<25 \mathrm{~Gy}$, and no constraints were applied to the lacrimal glands. Planning was optimized to minimized doses to OAR without compromising coverage of the PTV. VMAT and three non-coplanar IMRT (7-, 11-, and 15-beam) plans were compared using the heterogeneity and conformity indices $\left(\mathrm{HI}\right.$ and $\mathrm{Cl}$ ) of the PTV, $\mathrm{D}_{\max }$ and $\mathrm{D}_{\text {mean }}$ of the OAR, treatment delivery time, and monitor units (MUs).

Results: The HI and Cl of VMAT plan were superior to those of the 7-, 11-, and 15-beam non-coplanar IMRT. VMAT and non-coplanar IMRT (7-, 11-, and 15-beam) showed equivalent sparing effects for the optic nerves, optic chiasm, brainstem, and parotid glands. For the eyes and lenses, VMAT achieved equivalent or better sparing effects when compared with the non-coplanar IMRT plans. VMAT showed lower MUs and reduced treatment delivery time when compared with non-coplanar IMRT.
\end{abstract}

Conclusions: In 10 patients with nasal cavity or paranasal sinus cancer, a VMAT plan provided better homogeneity and conformity for PTV than non-coplanar IMRT plans, with a shorter treatment delivery time, while achieving equal or better OAR-sparing effects and using fewer MUs.

Keywords: Paranasal sinus cancer, Nasal cavity cancer, Volumetric modulated arc therapy, Non-coplanar intensity modulated radiotherapy, Planning target volume, Organs at risk

\footnotetext{
*Correspondence: Isw@amc.seoul.kr

Department of Radiation Oncology, Asan Medical Center, University of Ulsan

College of Medicine, 88, Olympic-ro-43-gil, Songpa-gu, Seoul 138-736, Republic of Korea
} 


\section{Background}

Nasal cavity and paranasal sinus cancers are relatively rare and account for about 5\% of head and neck cancers $[1,2]$. Most patients are diagnosed at an advanced stage, because symptoms and signs are usually nonspecific or asymptomatic in earlier stages. Standard treatment for locally advanced nasal cavity and paranasal sinus cancer is surgery followed by postoperative radiotherapy, or definitive radiotherapy with or without chemotherapy. In locally advanced stage, complete resection may not be possible due to the proximity to critical organs, and postoperative or definitive radiotherapy is needed. However, it is challenging to obtain optimal dose coverage for the target volume without compromising critical organs due to the proximity of critical organs such as eyes, optic nerves, optic chiasm, and brain. Intensitymodulated radiotherapy (IMRT) can provide more conformal dose coverage for the target volume, with reduced dosage to the adjacent critical organs, when compared with two- or three-dimensional radiotherapy [3-7]. Moreover, non-coplanar beams may provide additional optimization capacity within inverse planning processes for the target volumes of the nasal cavity and paranasal sinus cancer, which are usually located between both eyes. Recently, volumetric modulated arc therapy (VMAT) delivered by linear accelerator (LINAC) has become available. VMAT may reduce treatment delivery time with lower monitor units (MUs) while providing conformal dose distribution [8-11].

This study compares VMAT and non-coplanar IMRT for nasal cavity and paranasal sinus cancers with regard to the coverage of planning target volume (PTV) and the sparing of organs at risk (OAR).

\section{Methods}

\section{Patients and target volume}

Ten patients with nasal cavity or paranasal sinus cancers, who were previously treated with three-dimensional radiotherapy or IMRT between April 2011 and April 2013, were randomly selected from a clinical database maintained by the Asan Medical Center, Seoul, Korea. Treatment with VMAT (two-arc) and non-coplanar IMRT (7-, 11-, and 15-beam) was re-planned. Patient characteristics are shown in Table 1. All patients were T3-4NOM0 according to the American Joint Committee on Cancer 7th staging. Each patient was simulated with a thermoplastic mask to immobilize the head and neck. Computed tomography $(\mathrm{CT})$ images with a $2.5 \mathrm{~mm}$ slice thickness were obtained from vertex to clavicle. For patients previously treated with definitive radiotherapy, gross tumor volume (GTV) was defined as all gross disease on the magnetic resonance imaging (MRI), CT, or positron emission tomography (PET). Clinical target volumes (CTVs) included the nasal cavity, ethmoid sinus, frontal sinus, or maxillary sinus depending on the tumor location and the extent to cover microscopic spread (Table 1). To compensate for daily set-up variation and motion, the PTV was defined as the CTV plus a $5 \mathrm{~mm}$ margin. Contoured OAR were the eyes, lenses, optic nerves, optic chiasm, lacrimal glands, brainstem, brain, and parotid glands.

\section{Planning objectives}

The planning objectives were the same for VMAT and non-coplanar IMRT. The first objective was to deliver a prescribed dose of 60 Gy in 30 fractions to at least 95\% of the PTV. Second, both treatments were planned with a maximum dose $\left(D_{\max }\right)$ of $<50$ Gy to the optic nerves, optic chiasm, and brainstem, $<40$ Gy to the eyes and $<10$ Gy to the lenses. The planned mean dose $\left(D_{\text {mean }}\right)$ to the parotid glands was $<25 \mathrm{~Gy}$, and no constraint was applied to the lacrimal glands. Third, treatment planning aimed to reduce doses to OAR as much as possible without compromising the coverage of the PTV.

Priority of optimization was same as the order of planning objectives mentioned above. We put the highest priority to the maximum and minimum doses of PTV. For OARs, we put higher priority to the maximum doses of optic nerves, eyes, and lenses because these organs were very close to the PVT. After optimization, actual dose distributions in PTV and OARs were checked by dose statistics, dose volume histograms, and 3dimensional isodose lines whether planning objectives were met. If the optimization result was not acceptable, we modified optimization parameter such as priority and dose constraint and performed optimization again.

\section{VMAT planning}

For both VMAT and non-coplanar IMRT plans, $6 \mathrm{MV}$ photon beams were applied using a Varian TrueBeam STx (Varian Medical System, Palo Alto, CA), with multileaf collimators (MLCs) comprising 120 leaves of $2.5 \mathrm{~mm}$ width (in sliding window mode). Dose calculations were performed by Anisotropic Analytic Algorithm with a maximum dose rate of $600 \mathrm{MU} / \mathrm{min}$. VMAT plans were created using the two-arc technique, and optimization was performed using Eclipse progressive resolution optimization (version 10.0.28). The first arc ranged from $181^{\circ}$ to $179^{\circ}$ in clockwise rotation, and the second arc was from $179^{\circ}$ to $181^{\circ}$ in counter-clockwise rotation. The collimator angle was $30^{\circ}$ for the first arc and $330^{\circ}$ for the second arc.

\section{IMRT planning}

Eclipse dose volume optimization (version 10.0.28) was used for IMRT planning. To estimate the optimal number and angles of beams in non-coplanar IMRT plans for nasal cavity and paranasal sinus cancers, a beam angle optimization process was performed for 5 patients 


\section{Table 1 Patient characteristics}

\begin{tabular}{|c|c|c|c|c|}
\hline Case & Primary site & Stage $^{a}$ & Aim of radiotherapy & Clinical target volume \\
\hline 1 & Nasal cavity & T4aNOMO & Definitive & Nasal cavity, ethmoid, frontal sinus, ipsilateral maxillary sinus \\
\hline 2 & Nasal cavity & T4aNOMO & Definitive & Nasal cavity, ethmoid, frontal sinus, ipsilateral maxillary sinus \\
\hline 3 & Maxillary sinus & T4aNOMO & Definitive & Nasal cavity, ipsilateral maxillary sinus \\
\hline 4 & Maxillary sinus & T4aNOM0 & Postoperative & Nasal cavity, ethmoid, frontal sinus, ipsilateral maxillary sinus \\
\hline 5 & Nasal cavity & T4aNOMO & Postoperative & Nasal cavity, ethmoid, frontal sinus, ipsilateral maxillary sinus \\
\hline 6 & Ethmoid & T4bNOMO & Postoperative & Nasal cavity, ethmoid, frontal sinus, bilateral maxillary sinus \\
\hline 7 & Maxillary sinus & T3NOMO & Postoperative & Nasal cavity, ipsilateral maxillary sinus \\
\hline 8 & Nasal cavity & T4aNOMO & Definitive & Nasal cavity, ethmoid, frontal sinus, ipsilateral maxillary sinus \\
\hline 9 & Nasal cavity & T3NOMO & Postoperative & Nasal cavity, ethmoid, frontal sinus, bilateral maxillary sinus \\
\hline 10 & Nasal cavity & T4bN0M0 & Postoperative & Nasal cavity, ethmoid, frontal sinus, ipsilateral maxillary sinus \\
\hline
\end{tabular}

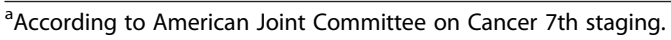

as an experimental purpose. The beam angle optimization was automatic process which is supported by Eclipse dose volume optimization (version 10.0.28). The optimal number of beams varied from 5 to 15 in each patient, and the beams were concentrated in the anterior direction, sparing both eyes and lenses. Based on these results, three noncoplanar IMRT (7-, 11-, and 15-beam) plans were developed for each patient. The 7-beam IMRT consisted of 5 coplanar beams at gantry angles of $0^{\circ}, 30^{\circ}, 100^{\circ}, 260^{\circ}$, and $330^{\circ}$, and 2 non-coplanar vertex beams at gantry angles of $30^{\circ}$ and $330^{\circ}$ with a $90^{\circ}$ couch rotation. The collimator angle was $30^{\circ}$ for the 2 coplanar beams at gantry angles of $30^{\circ}$ and $100^{\circ}, 330^{\circ}$ for the 2 coplanar beams at gantry angles of $260^{\circ}$ and $330^{\circ}$, and $0^{\circ}$ for the other beams. The 11-beam IMRT used 9 coplanar beams at gantry angles of $0^{\circ}, 20^{\circ}, 40^{\circ}, 60^{\circ}, 100^{\circ}, 260^{\circ}, 300^{\circ}, 320^{\circ}$, and $340^{\circ}$, and 2 non-coplanar vertex beams at gantry angles of $30^{\circ}$ and $330^{\circ}$ with a $90^{\circ}$ couch rotation. The collimator angle was $30^{\circ}$ for the 4 coplanar beams at gantry angles between $20^{\circ}$ and $100^{\circ}, 330^{\circ}$ for the 4 coplanar beams at gantry angles between $260^{\circ}$ and $340^{\circ}$, and $0^{\circ}$ for other beams. The 15 beam IMRT comprised 11 coplanar beams at gantry angles of $0^{\circ}, 15^{\circ}, 30^{\circ}, 45^{\circ}, 60^{\circ}, 100^{\circ}, 260^{\circ}, 300^{\circ}, 315^{\circ}, 330^{\circ}$ and $345^{\circ}$, and 4 non-coplanar vertex beams at gantry angles of $15^{\circ}, 30^{\circ}, 330^{\circ}$ and $345^{\circ}$ with a $90^{\circ}$ couch rotation. The collimator angle was $30^{\circ}$ for the 5 coplanar beams at gantry angles between $15^{\circ}$ and $100^{\circ}, 330^{\circ}$ for the 5 coplanar beams at gantry angles between $260^{\circ}$ and $345^{\circ}$, and $0^{\circ}$ for other beams.

\section{Plan evaluation and statistics}

PTV coverage was compared between VMAT and noncoplanar IMRT (7-, 11-, and 15-beam) using the heterogeneity index (HI) and conformity index (CI). The HI of PTV was defined as:

$\mathrm{HI}=\left(\mathrm{D}_{5 \%}-\mathrm{D}_{95 \%}\right) / \mathrm{D}_{\text {mean }}$

$\mathrm{D}_{5 \%}$ and $\mathrm{D}_{95 \%}$ are the minimum doses delivered to $5 \%$ and $95 \%$ volume of the PTV, and $\mathrm{D}_{\text {mean }}$ is the mean dose. A greater $\mathrm{HI}$ means higher heterogeneity. The $\mathrm{CI}$ of PTV was defined as:

$$
\mathrm{CI}=\mathrm{V}_{\mathrm{TV}} / \mathrm{V}_{\mathrm{PTV}}
$$

where $V_{\mathrm{TV}}$ is the treatment volume enclosed by the prescribed 60 Gy isodose surface, and $V_{\text {PTV }}$ is the volume of the PTV. A greater CI indicates lower conformity. To compare the OAR sparing of the VMAT and each of non-coplanar IMRT plans, $D_{\max }$ was evaluated for the eyes, lenses, optic nerves, optic chiasm, lacrimal glands, and the brainstem, and the $\mathrm{D}_{\text {mean }}$ of the parotid glands was recorded. Mean dose volume histograms (DVHs) for the PTV and OAR were calculated. MUs and treatment time for 2 Gy were also evaluated. We defined treatment delivery time in each patient as the sum of the beam on time with gantry rotation and beam setup time for VMAT plans and as the sum of the beam on time, beam setup time, and couch rotation time for non-coplanar IMRT plans, respectively. To estimate real treatment delivery time consisting of beam on time, beam setup time, and couch rotation time, VMAT and non-coplanar IMRT plans were delivered on Varian TrueBeam STx in quality assurance mode. Wilcoxon signed-rank tests were performed to compare the above parameters between the VMAT plan and each of the non-coplanar IMRT (7-, 11-, and 15-beam) plans. All statistical tests were two-sided and performed at the 5\% level of significance using SPSS (version 18.0).

\section{Results}

\section{Planning target volume coverage}

The HI and CI of VMAT and each of the 7-, 11-, and 15-beam non-coplanar IMRT plans for each of the 10 patients are shown in Figures 1 and 2. The HIs of the VMAT plan were better than those of the 7-, 11-, and 15-beam non-coplanar IMRT plans (mean HI 0.07 vs. 0.10 vs. 0.09 and 0.10 , respectively, $\mathrm{p}=0.004, \mathrm{p}=0.012$, and $\mathrm{p}=0.005$; Table 2). The CI was also superior in VMAT plans compared with each of 7-, 11-, 15-beam 


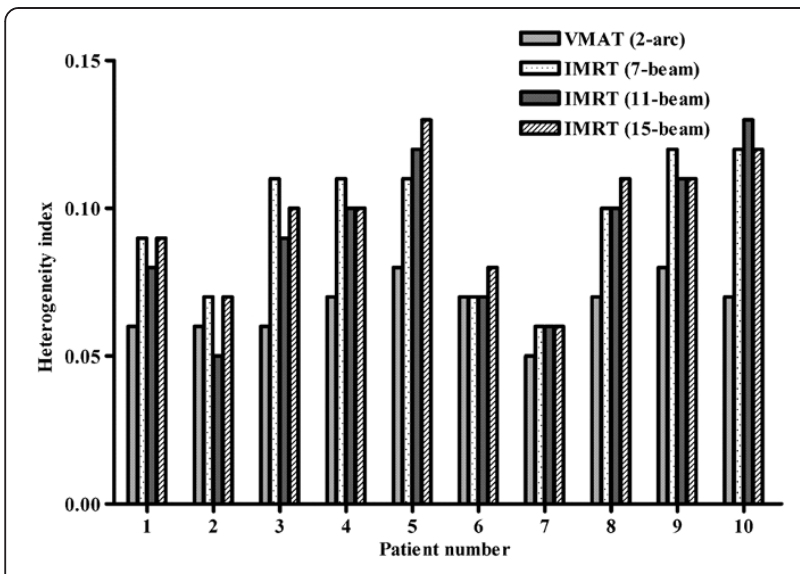

Figure 1 Comparison of the heterogeneity indices of volumetric modulated arc therapy (VMAT) and non-coplanar intensity modulated radiotherapy (IMRT; 7-, 11-, and 15-beam).

non-coplanar IMRT plans (mean CI, 1.05 vs. 1.13 vs. 1.10 and 1.10 , respectively, $\mathrm{p}=0.002, \mathrm{p}=0.008$, and $\mathrm{p}=$ 0.016) (Table 2). The mean DVHs for PTVs are shown in Figure 3.

\section{Dose to organs at risk}

The doses delivered to the various OAR by the VMAT plan and each of the 7-, 11-, and 15-beam non-coplanar IMRT plans for each of 10 patients are shown in Figure 4. The VMAT plan and the non-coplanar IMRT (7-, 11-, and 15-beam) plans showed equivalent sparing effects for the optic nerves, optic chiasm, brainstem, and parotid glands. For the eyes and lenses, VMAT achieved better or equivalent sparing effects when compared with each of the non-coplanar IMRT plans (Table 2). The maximum doses to the eyes of the VMAT plan were equivalent to those of the 11-beam non-coplanar IMRT plan (mean $D_{\max }$ of ipsilateral eye, 43.1 Gy vs. $46.1 \mathrm{~Gy}$,

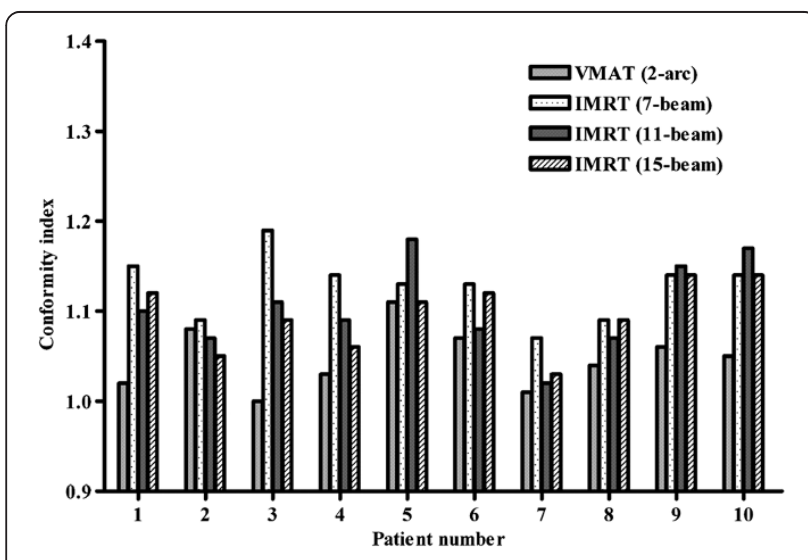

Figure 2 Comparison of the conformity indexes of volumetric modulated arc therapy (VMAT) and non-coplanar intensity modulated radiotherapy (IMRT; 7-, 11-, and 15-beam). $\mathrm{p}=0.064 ;$ mean $\mathrm{D}_{\max }$ of contralateral eye, 41.1 Gy vs. 42.7 Gy, $\mathrm{p}=0.492$ ), but lower than those of the 7-beam non-coplanar IMRT plan (mean $\mathrm{D}_{\max }$ of ipsilateral eye, 43.1 Gy vs. $48.8 \mathrm{~Gy}, \mathrm{p}=0.004$; mean $\mathrm{D}_{\max }$ of contralateral eye, 41.1 Gy vs. $44.7 \mathrm{~Gy}, \mathrm{p}=0.004)$. For lenses, VMAT had an equivalent sparing effect to the 7-beam non-coplanar IMRT plan (mean $\mathrm{D}_{\max }$ of ipsilateral lens, 10.5 Gy vs. $12.1 \mathrm{~Gy}, \mathrm{p}=0.193$; mean $\mathrm{D}_{\max }$ of contralateral lens, 10.2 Gy vs. 11.6 Gy, p=0.193) and a better sparing effect than 15-beam non-coplanar IMRT plan (mean $\mathrm{D}_{\max }$ of ipsilateral lens, 10.5 Gy vs. 13.6 Gy, p = 0.002; mean $\mathrm{D}_{\max }$ of contralateral lens, $10.2 \mathrm{~Gy}$ vs. $12.6 \mathrm{~Gy}, \mathrm{p}=0.006$ ). For lacrimal glands which no constraints were applied during treatment planning, each of the non-coplanar IMRT plans showed better $D_{\max }$ and $\mathrm{D}_{1 \%}$ than VMAT (Table 2). The mean DVHs for OAR are shown in Figure 3. Although DVHs for the optic chiasm, lenses, and lacrimal glands showed a similar trend to the $D_{\max }$, the DVHs for the optic nerves and eyes were better in non-coplanar IMRT, and the DVHs for brainstems were better in VMAT.

\section{MUs and treatment delivery times}

Mean MU was significantly lower in the VMAT plan than for each of 7-, 11-, 15-beam non-coplanar IMRT plans (485 vs. 2224 vs. 2350 and 2475 , respectively; $\mathrm{p}=0.002, \mathrm{p}=$ 0.002 , and $\mathrm{p}=0.002$ ). The mean beam on time was shorter in the VMAT plan than in the 7-, 11-, and 15-beam noncoplanar IMRT plans (2.48 minutes vs. 3.71 minutes vs. 3.92 minutes and 4.13 minutes, respectively; $\mathrm{p}=0.002, \mathrm{p}=$ 0.002 , and $\mathrm{p}=0.002$ ). The estimated beam setup time was 1.86 minutes in VMAT plans and 6.51 minutes, $10.23 \mathrm{mi}$ nutes, and 13.95 minutes in each of 7-, 11-, and 15-beam non-coplanar IMRT plans, respectively. In non-coplanar IMRT plans, the couch rotation time was estimated as 1.5 minutes. The entire treatment delivery time was estimated shorter in VMAT plan than in the 7-, 11-, and 15-beam non-coplanar IMRT plans (mean, 4.34 minutes vs. 11.72 minutes vs. 15.65 minutes and 19.58 minutes, respectively; $\mathrm{p}=0.002, \mathrm{p}=0.002$, and $\mathrm{p}=0.002$ ) (Table 2 ).

\section{Discussion}

In this study, the VMAT plan had better conformity and homogeneity with the PTV and equivalent to better sparing effects on OAR than the non-coplanar IMRT plans (7-, 11-, and 15- beam), for radiotherapy of nasal cavity and paranasal sinus cancers. The nasal cavity and paranasal sinus are adjacent to critical organs such as the eyes, optic nerves, optic chiasm, and brain. Optimizing dose coverage for the target volume without compromising critical organs is therefore a challenge in treatment planning. Previously, two-dimensional radiotherapy (2D-RT) with a weighted anterior field and two wedged lateral fields, and three-dimensional conformal 
Table 2 Dosimetric comparison for PTV, OARs, and MUs between VMAT and non-coplanar IMRT using 7-, 11-, and 15-beam

\begin{tabular}{|c|c|c|c|c|c|c|c|}
\hline & \multirow{2}{*}{$\begin{array}{c}\text { VMAT } \\
\text { Mean (SD) }\end{array}$} & \multirow{2}{*}{$\begin{array}{l}\text { 7-beam } \\
\text { IMRT } \\
\text { Mean (SD) }\end{array}$} & \multirow{2}{*}{$\begin{array}{l}\text { 11-beam } \\
\text { IMRT } \\
\text { Mean (SD) }\end{array}$} & \multirow{2}{*}{$\begin{array}{l}\text { 15-beam } \\
\text { IMRT } \\
\text { Mean (SD) }\end{array}$} & \multicolumn{3}{|c|}{$p$ value (VMAT vs.) } \\
\hline & & & & & 7-beam IMRT & 11-beam IMRT & 15-beam IMRT \\
\hline HI of PTV & $0.07(0.01)$ & $0.10(0.03)$ & $0.09(0.03)$ & $0.10(0.02)$ & $0.004^{*}$ & $0.012^{*}$ & $0.005^{*}$ \\
\hline Cl of PTV & $1.05(0.03)$ & $1.13(0.03)$ & $1.10(0.05)$ & $1.10(0.04)$ & $0.002^{*}$ & $0.008^{*}$ & $0.016^{*}$ \\
\hline Ipsilateral optic nerve $D_{\max }(G y)$ & $47.9(2.16)$ & $46.9(3.15)$ & $46.3(3.21)$ & $46.0(3.91)$ & 0.492 & 0.492 & 0.193 \\
\hline$D_{1 \%}(G y)$ & $45.7(2.36)$ & $44.1(2.95)$ & $43.0(2.40)$ & $43.1(3.17)$ & 0.322 & 0.084 & 0.064 \\
\hline Contralateral optic nerve $D_{\max }$ (Gy) & $47.0(2.86)$ & $46.0(2.86)$ & $46.8(2.79)$ & $46.0(2.87)$ & 0.275 & 0.695 & 0.322 \\
\hline$D_{1 \%}(G y)$ & $45.3(2.65)$ & $43.1(2.79)$ & $44.2(2.21)$ & $43.6(2.55)$ & 0.129 & 0.275 & 1.000 \\
\hline Optic chiasm $D_{\max }(G y)$ & $46.7(2.64)$ & $45.3(3.46)$ & $45.6(2.67)$ & $45.0(2.87)$ & 0.275 & 0.322 & 0.193 \\
\hline$D_{1 \%}(G y)$ & $46.6(2.48)$ & $42.7(3.01)$ & $42.9(2.45)$ & $42.5(2.96)$ & 0.193 & 0.166 & 0.131 \\
\hline Brainstem $D_{\max }(G y)$ & $44.2(3.31)$ & $46.0(2.33)$ & $44.5(2.45)$ & $45.1(2.24)$ & 0.105 & 0.770 & 0.322 \\
\hline$D_{1 \%}(G y)$ & $39.8(3.05)$ & $41.7(1.77)$ & $40.9(1.77)$ & $41.8(1.62)$ & 0.160 & 0.359 & 0.064 \\
\hline Ipsilateral eye $D_{\max }(G y)$ & $43.1(3.56)$ & $48.8(4.51)$ & $46.1(4.59)$ & $45.9(3.30)$ & $0.004^{*}$ & 0.064 & $0.037^{*}$ \\
\hline$D_{1 \%}(G y)$ & $38.8(3.45)$ & $39.8(3.89)$ & $37.6(3.36)$ & $37.4(2.56)$ & 0.322 & 0.322 & 0.275 \\
\hline Contralateral eye $D_{\max }(G y)$ & $41.1(3.15)$ & $44.7(2.94)$ & $42.7(4.37)$ & $42.4(3.86)$ & $0.004^{*}$ & 0.492 & 0.322 \\
\hline$D_{1 \%}(G y)$ & $36.7(3.70)$ & $35.7(2.89)$ & $34.4(4.03)$ & $33.9(3.84)$ & 0.264 & 0.078 & 0.064 \\
\hline Ipsilateral lens $D_{\max }(G y)$ & $10.5(1.36)$ & $12.1(2.28)$ & $12.6(1.99)$ & $13.6(1.79)$ & 0.193 & $0.020^{*}$ & $0.002^{*}$ \\
\hline$D_{1 \%}(G y)$ & $9.77(1.19)$ & $11.9(2.32)$ & $12.2(2.10)$ & $13.3(1.96)$ & $0.014^{*}$ & $0.006^{*}$ & $0.002^{*}$ \\
\hline Contralateral lens $D_{\max }(G y)$ & $10.2(1.17)$ & $11.6(2.18)$ & $11.8(2.19)$ & $12.6(2.12)$ & 0.193 & 0.084 & $0.006^{*}$ \\
\hline$D_{1 \%}(G y)$ & $9.49(0.93)$ & $11.2(2.36)$ & $11.4(2.26)$ & $12.2(2.29)$ & 0.053 & $0.012^{*}$ & $0.04^{*}$ \\
\hline Ipsilateral lacrimal gland $D_{\max }$ (Gy) & $28.5(10.3)$ & $15.2(6.37)$ & $18.1(9.93)$ & $16.5(6.27)$ & $0.004^{*}$ & $0.004^{*}$ & $0.002^{*}$ \\
\hline $\mathrm{D}_{1 \%}(\mathrm{~Gy})$ & $27.1(1.02)$ & $14.1(5.50)$ & $17.0(9.41)$ & $15.4(5.71)$ & $0.002^{*}$ & $0.004^{*}$ & $0.002^{*}$ \\
\hline Contralateral lacrimal gland $D_{\max }(G y)$ & $22.1(4.44)$ & $11.4(6.06)$ & $12.4(6.02)$ & $11.0(5.27)$ & $0.002^{*}$ & $0.002^{*}$ & $0.002^{*}$ \\
\hline$D_{1 \%}(G y)$ & $21.0(4.69)$ & $10.8(5.77)$ & $11.3(5.74)$ & $10.4(5.17)$ & $0.002^{*}$ & $0.002^{*}$ & $0.002^{*}$ \\
\hline Ipsilateral parotid gland $D_{\text {mean }}(G y)$ & $12.5(9.91)$ & $12.2(8.75)$ & $12.7(8.93)$ & $11.8(8.45)$ & 0.922 & 0.625 & 0.625 \\
\hline Contralateral parotid gland $D_{\text {mean }}(G y)$ & $9.84(7.80)$ & $8.84(6.87)$ & $9.27(6.80)$ & $8.07(5.87)$ & 0.131 & 0.492 & 0.084 \\
\hline Monitor units & $485(66.3)$ & $2224(423)$ & $2350(451)$ & $2475(384)$ & $0.002^{*}$ & $0.002^{*}$ & $0.002^{*}$ \\
\hline Treatment delivery time (Minutes) & $4.34(0.00)$ & $11.72(0.71)$ & $15.65(0.75)$ & $19.58(0.64)$ & $0.002^{*}$ & $0.002^{*}$ & $0.002^{*}$ \\
\hline
\end{tabular}

${ }^{*} p$ value $<0.05$.

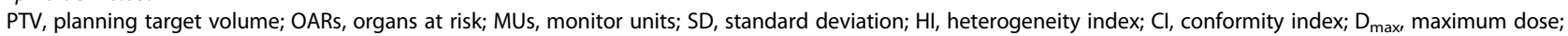
$\mathrm{D}_{\text {mean, }}$ mean dose; $\mathrm{D}_{1 \%}$, minimum dose delivered $1 \%$ of the PTV.

radiotherapy (3D-CRT) were used to avoid both eyes and to overcome dose heterogeneity. However, the complex shape and tissue heterogeneity of the target volume negatively affect dose distribution, and treatment outcomes were unsatisfactory in terms of local control and optic pathway preservation [7]. With the development of computerized optimization processes, techniques with more conformal and homogeneous dose distributions have become possible. These include IMRT, a form of 3D-CRT able to generate non-uniform intensity using beamlets. Several studies have compared IMRT to 2DRT or 3D-CRT for the treatment of nasal cavity and paranasal sinus cancer [3-7]. Lee et al. compared IMRT using the complementary boost-fields with 3D-CRT for ethmoid sinus cancer [4]. The homogeneity of the PTV was higher for IMRT than for 3D-CRT, and OAR sparing was similar. Other studies also reported that IMRT improved PTV coverage and/or OAR sparing [3,5-7]. However, IMRT needs more MUs and longer treatment delivery time than 2D-RT or 3D-CRT, and it has inevitable disadvantages. Wang et al. evaluated the impact of prolonged treatment delivery time on tumor control in a biologic model, and calculated that cell killing was decreased with prolonged treatment delivery time, especially in tumors with low alpha/beta ratios or short repair half-times [12]. Although there are no clinical studies to date, several studies have shown the detrimental effect of prolonged treatment delivery time using cell lines or xenograft models [13-17].

VMAT allows rapid delivery of inverse planned radiotherapy by continuous gantry rotation and simultaneous modulation of MLCs, and has recently become available. 


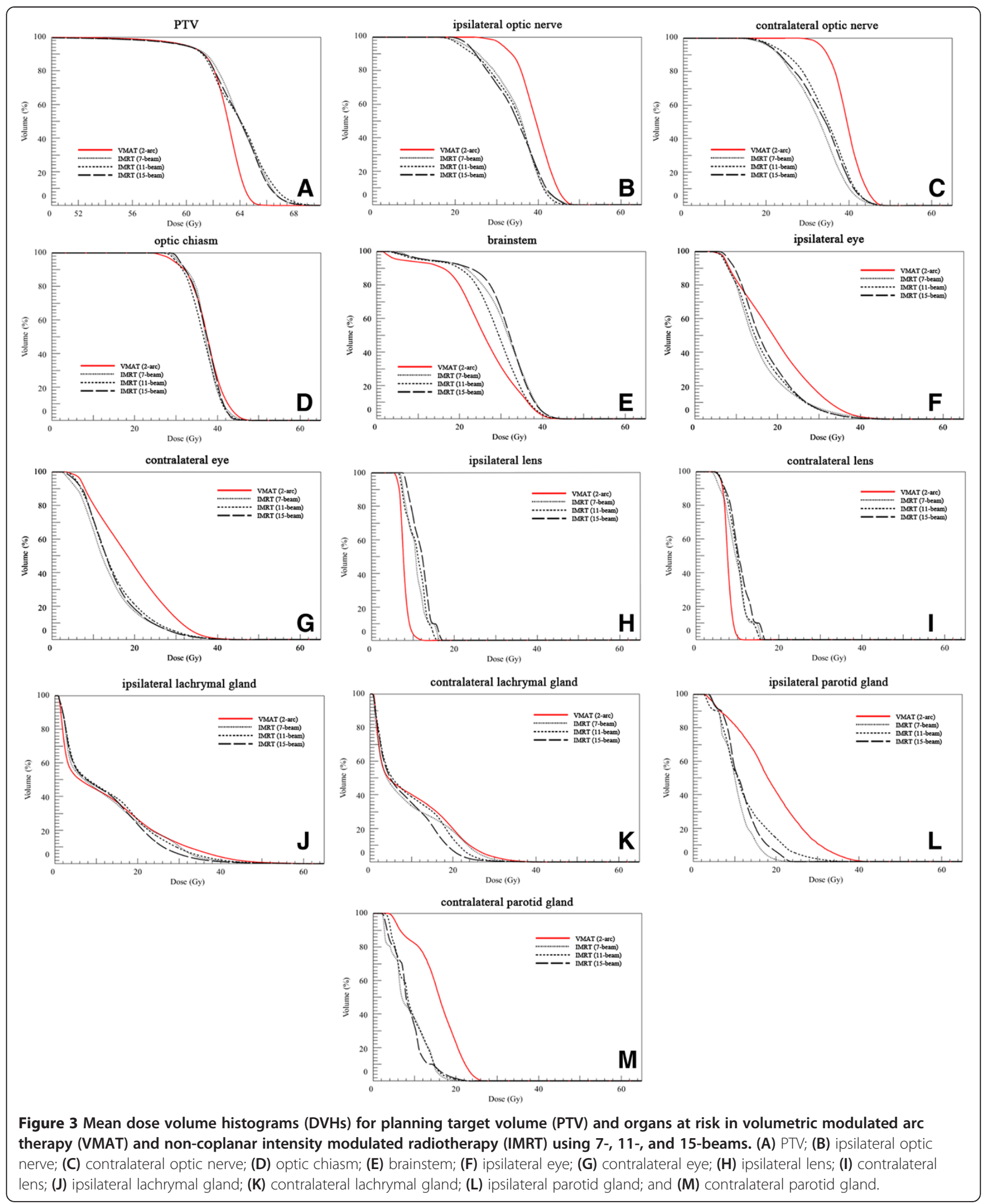

Several studies have compared VMAT with fixed beam IMRT in terms of dose coverage, OAR-sparing effects, reduced treatment delivery time and decreased MUs
[8-11]. In these studies, VMAT showed equivalent or better PTV coverage and OAR-sparing effects, decreased treatment delivery time and fewer MUs [8-11]. 


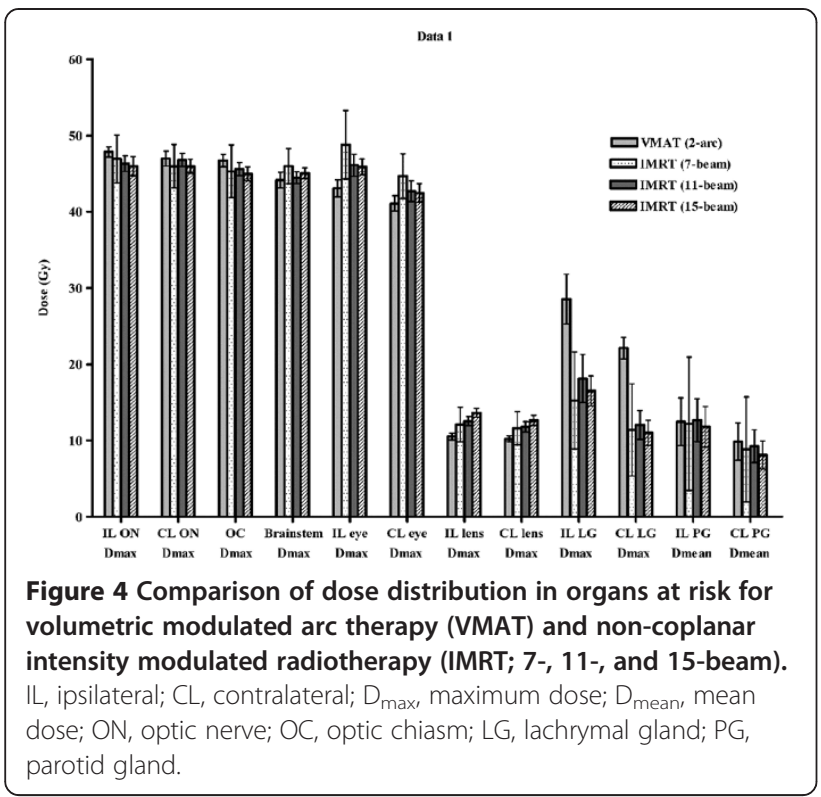

Studies of VMAT have been conducted for nasal cavity and paranasal sinus cancers, which are usually located between both eyes and have complex shaped target volumes [18-21]. The dosimetric superiority of VMAT for PTV coverage and OAR sparing shown in the present study are consistent with most previous studies. Sankaralingam et al. compared VMAT (two-arc) and coplanar IMRT (5-beam, sagittal) in nasal cavity and paranasal sinus cancer $(\mathrm{n}=5)$ and reported that VMAT showed better homogeneity in all patients and better conformity in 3 of 5 patients. However, the number of patients was too small to establish statistically significant differences [19]. In another study, step-and-shoot IMRT was compared with single arc and multiple arc VMAT for localized prostate cancers $(n=5)$, pharyngeal cancers $(n=$ 10), and paranasal sinus cancers $(n=5)$ [18]. VMAT showed equivalent or improved PTV coverage, homogeneity, conformity, and OAR sparing when compared with step-and-shoot IMRT in localized prostate cancers and pharyngeal cancers, but not in paranasal sinus cancer patients, where increased doses to the lenses were observed in VMAT using multiple arcs. More recently, Nguyen et al. reported a dosimetric comparative study which compared VMAT (3-arc) and IMRT (9-beam) for nasal cavity cancer $(\mathrm{n}=10)$ [20]. In that study, VMAT showed comparable PTV coverage and equivalent or better reduced dose to the OARs as well as fewer MUs and shorter treatment delivery times. Similarly, VMAT showed equivalent PTV coverage to IMRT with decreased delivery times and MUs in 20 patients with head and neck cancers [21]. It is difficult to compare these studies directly because of differences in the target volumes, prescribed doses, number of prescribed dose levels, planning algorithm, plan evaluation methods, and small numbers of patients. However, VMAT shows better or equivalent PTV coverage and OAR-sparing effects in all studies except that of Guckenberger et al.

VMAT has greater freedom for optimization when compared with IMRT, as it allows gantry rotation with variable speed during treatment delivery, simultaneous MLC modification and simultaneous dose rate variation [22-25]. However, in nasal cavity and paranasal sinus cancer patients, whose target volumes are usually located between the eyes, the benefits of gantry rotation may be limited due to the relative limitations on beam angle selection. This could increase low-dose 'tails' to the lenses, and may account for the observations in Guckenberger et al. [18]. The present study evaluated whether the optimization freedom benefits of VMAT were realizable in nasal cavity or paranasal sinus cancers, or whether non-coplanar IMRT remained superior for PTV coverage and OAR sparing in that area. Three noncoplanar IMRT (7-, 11-, and 15-beam) plans were implemented for each patient, none of which were superior to VMAT in PTV coverage. All three IMRT plans had greater $\mathrm{D}_{\max }$ and $\mathrm{D}_{\text {mean }}$, and worse mean $\mathrm{DVH}$, for both lenses. Non-coplanar, multi-beam fixed beam IMRT may not therefore overrule the optimization freedom of VMAT, even if the number and angle of beams are optimal. Moreover, in the present study, beams from the posterior direction were the most limited because of the brainstem and brain. The superiority of the VMAT plan might also arise from this limitation of the posterior beams in the non-coplanar IMRT plans, but further studies using more posterior direction beams are needed to confirm this suggestion.

This study has several limitations. First, it is a planning study comparing the dosimetric parameters between non-coplanar IMRT and VMAT. Local control and toxicities can be influenced by various factors such as past medical history, histologic types, and combination with chemotherapy as well as by dosimetric parameters. Whether the superiority of VMAT can be translated into clinical benefit is therefore not clear. Several studies have reported that dosimetric improvement from 2D-RT or 3D-CRT to IMRT resulted in clinical benefit with respect to local control and toxicities, and technical advances within IMRT may also result in clinical improvements [7,26-28]. However, the relative dosimetric superiority of VMAT to IMRT may be smaller than that of IMRT to two- or three-dimensional radiotherapy, and may not result in clinical difference. Moreover, in present planning study, there is also a general weakness that results may not be consistent when the calculation and/or optimization algorithms are changed. Second, the two-arc approach was used for VMAT planning whereas 7-, 11-, and 15-beam IMRT was planned. A single arc VMAT plan can be sufficient for areas with less complex 
shapes and single-dose prescription levels [18,29-31]. However, for complex target areas or multiple dose prescription levels, the two-arc technique was needed to achieve PTV coverage and OAR sparing better than or equivalent to fixed beam IMRT, and single arc VMAT was inferior to fixed beam IMRT [10,11,18,32,33]. Nasal cavity and paranasal sinus cancers have complex target volume shapes, and so two-arc VMAT was chosen for the current study. Two-arc VMAT was equivalent to or better than non-coplanar IMRT in this setting. However, further studies of the details of VMAT such as the gantry rotation angle, number of arcs, and using noncoplanar arcs are needed. Third, the current study used a single-dose prescription level. The differences between VMAT and non-coplanar IMRT when performing simultaneous integrated boost techniques with multiple dose prescription level was not evaluated, and further studies are needed. Fourth, present study did not reflect the difference in the treatment delivery error between VMAT and non-coplanar IMRT. Although there has been no definite conclusion about the influence of delivery error yet, random and systematic errors of MLC position, gantry position, and MU are present in both VMAT and non-coplanar IMRT plans. Furthermore, couch rotation errors and intrafraction setup errors are also present in non-coplanar IMRT plans. Despite these shortcomings, the present study is one of a small number comparing VMAT with IMRT for nasal cavity and paranasal sinus cancers, and was performed in a relatively large number of patients. Attempts were made to reduce bias in several ways. First, all patients were re-planned for VMAT and non-coplanar IMRT, instead of using previous plans. Second, the same planning objectives were applied for VMAT and non-coplanar IMRT. Third, dose calculations were performed using the Anisotropic Analytic Algorithm. Furthermore, the MLC with $2.5 \mathrm{~mm}$ width in the Varian TrueBeam STx apparatus used in this study enables more precise plans for the complex shaped target volumes of nasal cavity and paranasal sinus cancers.

\section{Conclusions}

In patients with nasal cavity or paranasal sinus cancer, VMAT provided better homogeneity and conformity for PTV than 7-, 11-, and 15- beam non-coplanar IMRT plans, while achieving equal or better OAR-sparing effects, using fewer MUs and shortening treatment delivery times. VMAT can therefore be considered a good treatment option for nasal cavity and paranasal sinus cancers.

\section{Abbreviations}

VMAT: Volumetric modulated arc therapy; IMRT: Intensity modulated radiotherapy; PTV: Planning target volume; OAR: Organ at risk; $D_{\text {max }}$ Maximum dose; $D_{\text {mean: }}$ Mean dose; HI: Heterogeneity index; Cl: Conformity index; MUs: Monitor units; LINAC: Linear accelerator; CT: Computed tomography; GTV: Gross tumor volume; MRI: Magnetic resonance imaging; PET: Positron emission tomography; CTV: Clinical target volumes; MLCs: Multi-leaf collimators; D $_{5 \%}$ : Minimum doses delivered to 5\% volume of the PTV; $D_{95 \%}$ : Minimum doses delivered to $95 \%$ volume of the PTV; DVHs: Dose volume histograms; 2D-RT: Two-dimensional radiotherapy; 3D-CRT: Three-dimensional conformal radiotherapy.

\section{Competing interests}

The authors declare that they have no competing interests.

\section{Authors' contributions}

Each author had participated sufficiently in the work to take public responsibility for appropriate portions of the content. YJ and SWL participated in research design, coded the patient database, conducted the analysis and wrote the manuscript draft and revised the manuscript. JK participated in planning process of IMRT and VMAT and in coding of the database. IC analyzed the data and provided support for figure draft. SMY, JHK, JHP, SYS, YSK, SSK, and JHJ contributed to conception and study design. EKC and SDA provided additional guidance and support for this research. All authors read and approved the final manuscript.

\section{Acknowledgements}

No outside funding or grants to be declared.

Received: 26 March 2014 Accepted: 9 August 2014

Published: 30 August 2014

\section{References}

1. Roush GC: Epidemiology of cancer of the nose and paranasal sinuses: current concepts. Head Neck Surg 1979, 2(1):3-11.

2. Muir CS, Nectoux J: Descriptive epidemiology of malignant neoplasms of nose, nasal cavities, middle ear and accessory sinuses. Clin Otolaryngol Allied Sci 1980, 5(3):195-211.

3. Adams EJ, Nutting CM, Convery DJ, Cosgrove VP, Henk JM, Dearnaley DP, Webb S: Potential role of intensity-modulated radiotherapy in the treatment of tumors of the maxillary sinus. Int J Radiat Oncol Biol Phys 2001, 51(3):579-588.

4. Lee SW, Kim GE, Suh CO, Chu SS, Lee KK, Moon SR: Intensity modulation technique using the complementary boost-fields for ethmoid sinus cancer. Clin Oncol (R Coll Radiol) 2002, 14(3):241-249.

5. O'Daniel JC, Dong L, Kuban DA, Liu H, Schechter N, Tucker SL, Rosen I: The delivery of IMRT with a single physical modulator for multiple fields: a feasibility study for paranasal sinus cancer. Int J Radiat Oncol Biol Phys 2004, 58(3):876-887.

6. Huang D, Xia P, Akazawa P, Akazawa C, Quivey JM, Verhey LJ, Kaplan M, Lee N: Comparison of treatment plans using intensity-modulated radiotherapy and three-dimensional conformal radiotherapy for paranasal sinus carcinoma. Int J Radiat Oncol Biol Phys 2003, 56(1):158-168.

7. Chi A, Nguyen NP, Tse W, Sobremonte G, Concannon P, Zhu A: Intensity modulated radiotherapy for sinonasal malignancies with a focus on optic pathway preservation. I Hematol Oncol 2013, 6:4

8. Shaffer R, Nichol AM, Vollans E, Fong M, Nakano S, Moiseenko V, Schmuland M, Ma R, McKenzie M, Otto K: A comparison of volumetric modulated arc therapy and conventional intensity-modulated radiotherapy for frontal and temporal high-grade gliomas. Int J Radiat Oncol Biol Phys 2010, 76(4):1177-1184.

9. Johnston M, Clifford S, Bromley R, Back M, Oliver L, Eade T: Volumetricmodulated arc therapy in head and neck radiotherapy: a planning comparison using simultaneous integrated boost for nasopharynx and oropharynx carcinoma. Clin Oncol (R Coll Radiol) 2011, 23(8):503-511.

10. Verbakel WF, Cuijpers JP, Hoffmans D, Bieker M, Slotman BJ, Senan S: Volumetric intensity-modulated arc therapy vs. conventional IMRT in head-and-neck cancer: a comparative planning and dosimetric study. Int J Radiat Oncol Biol Phys 2009, 74(1):252-259.

11. Vanetti E, Clivio A, Nicolini G, Fogliata A, Ghosh-Laskar S, Agarwal JP, Upreti RR, Budrukkar A, Murthy V, Deshpande DD, Shrivastava SK, Dinshaw KA, Cozzi L: Volumetric modulated arc radiotherapy for carcinomas of the oro-pharynx, hypo-pharynx and larynx: a treatment planning comparison with fixed field IMRT. Radiother Oncol 2009, 92(1):111-117.

12. Wang JZ, Li XA, D'Souza WD, Stewart RD: Impact of prolonged fraction delivery times on tumor control: a note of caution for intensity-modulated radiation therapy (IMRT). Int J Radiat Oncol Biol Phys 2003, 57(2):543-552. 
13. Jiang $L$, Xiong $X P, H u C S, O u Z L$, Zhu GP, Ying HM: In vitro and in vivo studies on radiobiological effects of prolonged fraction delivery time in A549 cells. J Radiat Res 2013, 54(2):230-234.

14. Moiseenko V, Banath JP, Duzenli C, Olive PL: Effect of prolonging radiation delivery time on retention of gammaH2AX. Radiat Oncol 2008, 3:18.

15. Mu X, Lofroth PO, Karlsson M, Zackrisson B: The effect of fraction time in intensity modulated radiotherapy: theoretical and experimental evaluation of an optimisation problem. Radiother Oncol 2003, 68(2):181-187.

16. Wang $X$, Xiong XP, Lu J, Zhu GP, He SQ, Hu CS, Ying HM: The in vivo study on the radiobiologic effect of prolonged delivery time to tumor control in C57BL mice implanted with Lewis lung cancer. Radiat Oncol 2011, 6:4

17. Zheng XK, Chen $\mathrm{LH}$, Yan X, Wang HM: Impact of prolonged fraction dose-delivery time modeling intensity-modulated radiation therapy on hepatocellular carcinoma cell killing. World J Gastroenterol 2005, 11(10):1452-1456.

18. Guckenberger M, Richter A, Krieger T, Wilbert J, Baier K, Flentje M: Is a single arc sufficient in volumetric-modulated arc therapy (VMAT) for complex-shaped target volumes? Radiother Oncol 2009, 93(2):259-265.

19. Sankaralingam M, Glegg M, Smith S, James A, Rizwanullah M: Quantitative comparison of volumetric modulated arc therapy and intensity modulated radiotherapy plan quality in sino-nasal cancer. J Med Phys 2012, 37(1):8-13.

20. Nguyen K, Cummings D, Lanza VC, Morris K, Wang C, Sutton J, Garcia J: A dosimetric comparative study: volumetric modulated arc therapy vs intensity-modulated radiation therapy in the treatment of nasal cavity carcinomas. Med Dosim 2013, 38(3):225-232.

21. Studenski MT, Bar-Ad V, Siglin J, Cognetti D, Curry J, Tuluc M, Harrison AS: Clinical experience transitioning from IMRT to VMAT for head and neck cancer. Med Dosim 2013, 38(2):171-175.

22. Otto K: Volumetric modulated arc therapy: IMRT in a single gantry arc Med Phys 2008, 35(1):310-317.

23. Otto K: Letter to the Editor on 'Single-Arc IMRT?' Phys Med Biol 2009, 54(8):L37-41. author reply L43-34.

24. Nicolini G, Clivio A, Cozzi L, Fogliata A, Vanetti E: On the impact of dose rate variation upon RapidArc implementation of volumetric modulated are therapy. Med Phys 2011, 38(1):264-271.

25. Vanetti E, Nicolini G, Nord J, Peltola J, Clivio A, Fogliata A, Cozzi L: On the role of the optimization algorithm of $\operatorname{Rapid} \operatorname{Arc}((\mathrm{R}))$ volumetric modulated arc therapy on plan quality and efficiency. Med Phys 2011, 38(11):5844-5856.

26. Chen AM, Daly ME, Bucci MK, Xia P, Akazawa C, Quivey JM, Weinberg V, Garcia J, Lee NY, Kaplan MJ, El-Sayed I, Eisele DW, Fu KK, Phillips TL: Carcinomas of the paranasal sinuses and nasal cavity treated with radiotherapy at a single institution over five decades: are we making improvement? Int J Radiat Oncol Biol Phys 2007, 69(1):141-147.

27. Dirix P, Vanstraelen B, Jorissen M, Vander Poorten V, Nuyts S: Intensitymodulated radiotherapy for sinonasal cancer: improved outcome compared to conventional radiotherapy. Int J Radiat Oncol Biol Phys 2010, 78(4):998-1004.

28. Al-Mamgani A, Monserez D, Rooij P, Verduijn GM, Hardillo JA, Levendag PC: Highly-conformal intensity-modulated radiotherapy reduced toxicity without jeopardizing outcome in patients with paranasal sinus cancer treated by surgery and radiotherapy or (chemo)radiation. Oral Oncol 2012, 48(9):905-911.

29. Palma D, Vollans E, James K, Nakano S, Moiseenko V, Shaffer R, McKenzie M, Morris J, Otto K: Volumetric modulated arc therapy for delivery of prostate radiotherapy: comparison with intensity-modulated radiotherapy and three-dimensional conformal radiotherapy. Int J Radiat Oncol Biol Phys 2008, 72(4):996-1001.

30. Cozzi L, Dinshaw KA, Shrivastava SK, Mahantshetty U, Engineer R, Deshpande DD, Jamema SV, Vanetti E, Clivio A, Nicolini G, Fogliata A: A treatment planning study comparing volumetric arc modulation with RapidArc and fixed field IMRT for cervix uteri radiotherapy. Radiother Oncol 2008, 89(2):180-191.

31. Fogliata A, Clivio A, Nicolini G, Vanetti E, Cozzi L: Intensity modulation with photons for benign intracranial tumours: a planning comparison of volumetric single arc, helical arc and fixed gantry techniques. Radiother Oncol 2008, 89(3):254-262.
32. Lee TF, Ting HM, Chao PJ, Fang FM: Dual arc volumetric-modulated arc radiotherapy (VMAT) of nasopharyngeal carcinomas: a simultaneous integrated boost treatment plan comparison with intensity-modulated radiotherapies and single arc VMAT. Clin Oncol (R Coll Radiol) 2012, 24(3):196-207

33. Clivio A, Fogliata A, Franzetti-Pellanda A, Nicolini G, Vanetti E, Wyttenbach $R$ Cozzi L: Volumetric-modulated arc radiotherapy for carcinomas of the anal canal: A treatment planning comparison with fixed field IMRT. Radiother Oncol 2009, 92(1):118-124.

doi:10.1186/1748-717X-9-193

Cite this article as: Jeong et al:: A dosimetric comparison of volumetric modulated arc therapy (VMAT) and non-coplanar intensity modulated radiotherapy (IMRT) for nasal cavity and paranasal sinus cancer. Radiation Oncology 2014 9:193.

\section{Submit your next manuscript to BioMed Central and take full advantage of:}

- Convenient online submission

- Thorough peer review

- No space constraints or color figure charges

- Immediate publication on acceptance

- Inclusion in PubMed, CAS, Scopus and Google Scholar

- Research which is freely available for redistribution

Submit your manuscript at www.biomedcentral.com/submit
C Biomed Central 